\title{
Announcing our new journal: International Cancer Conference Journal (ICCJ)
}

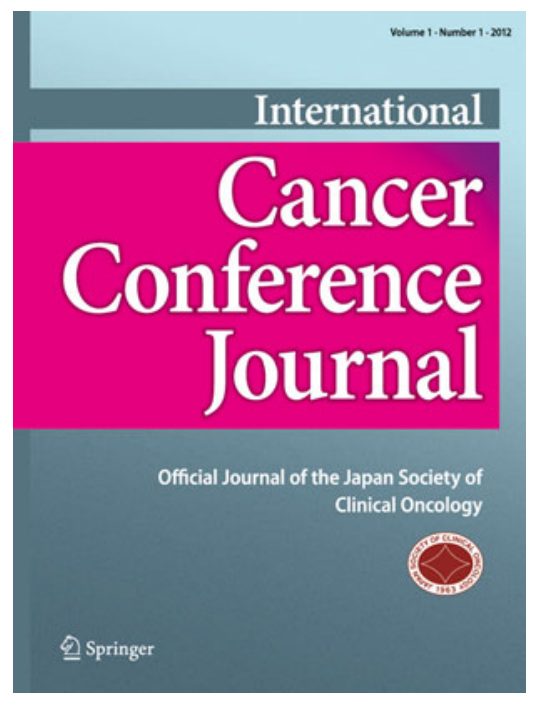

The Japan Society of Clinical Oncology produces an official journal, the International Journal of Clinical Oncology (IJCO). It is published in English, is widely indexed, and now has an impact factor of 1.508. Every day we receive many original articles submitted for publication in IJCO, but owing to page limitations, we must forgo publication of many good papers, including case reports.

Recently, however, information about clinical oncology in areas such as cancer surgery, chemotherapy, radiotherapy, and palliative care for cancer patients has received much attention, increasing the need for knowledge of case reports, comprehensive reviews, and cancer board conferences. We know that clinical experience about one patient can change the world of medical science, and therefore it is still important to publish case reports.

For that reason, we have now decided to launch a new journal, the International Cancer Conference Journal
(ICCJ), to accept excellent case reports, thereby contributing to clinical education and discussion. This is a unique, online journal, providing benefits such as quick review and publication, along with free color presentation of figures and videos. Additionally, in the future, readers will be able to participate in the discussions through letters and commentary in the journal's cancer board conferences.

On behalf of the Japan Society of Clinical Oncology, we sincerely look forward to your submission of cancer case reports and other contributions to the International Cancer Conference Journal (ICCJ).

Journal title: International Cancer Conference Journal Editor-in-chief: Yoshiharu Sakai, MD

Published format: Electronic online edition only

Frequency of publication: 4 times per year (issued quarterly)

Contents: Clinical Reviews or Cancer Board Conferences, 1 or 2 per issue; Case Reports, approximately 10 per issue Initial publication: January 2012

Submission and publication cost: Free of charge, including color pages

Submission guidance: Use the online system Editorial Manager starting in May 2011

[Inquiries]

c/o Invention Center of the Kinki Districts (Kinki Chiho Hatsumei Center), 14 Yoshidakawaramachi, Sakyo-ku, Kyoto 606-8305, Japan

Fax +81-75-761-9724

E-mail: jsco@gakkai.net

International Journal of Clinical Oncology

Editor-in-Chief

Ikuo Konishi, MD 